\title{
3 Research Suare \\ Case Report of Angiocentric Glioma Lacking Epilepsy and Discuss Its Relationship With Epilepsy
}

\author{
HaiLun Cheng \\ Peking University Shenzhen Hospital \\ WeiHua Yin ( $\nabla$ weihuayin_sz@sina.com ) \\ Peking University Shenzhen Hospital \\ QinQin Xu \\ Peking University Shenzhen Hospital \\ XiaoXin Shi \\ Peking University Shenzhen Hospital
}

Case Report

Keywords: AG, epilepsy, MYB-QKI, immunohistochemistry

Posted Date: March 1st, 2022

DOI: https://doi.org/10.21203/rs.3.rs-1374877/v1

License: (c) (1) This work is licensed under a Creative Commons Attribution 4.0 International License.

Read Full License 


\section{Abstract}

Background: Angiocentric glioma is a rare neuro tumor, the general public believes that the typical clinical feature of angiocentric glioma (AG) is a history of epilepsy. This case have unique research value because it is lack history of epilepsy, it is provide evidence to exploration of the causes of AG-related epilepsy symptoms.

Case presentations: A 16-year-old boy came to the hospital because of dizziness and blurred vision. The patient's CT stan detected a solid mass in the right frontotemporal lobe. Histologic finding the tumor is predominantly composed of elongated tumor cells forming rings around the blood vessels, the immunohistochemical staining show GFAPID2-40匹S100־Vimentin have positive expression, and Ki-67 proliferation index is 1\% 5\%, FISH-tissue show MYB gene rearrangement detection. According these evidences, this case was diagnosed as angiocentric glioma. the patient's physical condition was stable and there was no recurrence after the operation.

Conclusion: We believe that MYB gene break rearrangement is related to the cause of AG and MYB-QKI gene rearrangement may be one of the important causes of $A G$, but it is not directly related to AG-related epilepsy.

\section{Background}

angiocentric glioma(AG) is a rare neuro tumor, and its research progress is slow due to fewer cases. The vast majority of angiocentric glioma cases reported so far have a history of epilepsy. The general public believes that the typical clinical feature of AG is a history of epilepsy[1]. AG was proposed by Wang and Lellouch-Tubiana in 2005 [2] and was included in the WHO central nervous system tumors in 2007 among other types of neuroepithelial tumors. It is determined by histopathology. Characteristic naming: namely, vascular central growth, monomorphic bipolar tumor cells, and ependymal differentiation, and its lesions are equivalent to $\mathrm{WHO}$ I grade[1].

\section{Case Report}

A 16-year-old boy came to the hospital because of atypical dizziness and blurred vision. The patient's CT detected a solid, well-defined mass in the right frontotemporal lobe(Fig. 1). The clinically suspected glioma was highly suspected within a week. Later surgery was performed. Pathologically, the boundary between the spindle cell tumor cells and the surrounding brain tissue was unclear, and the tumor cells were diffusely distributed in a sheet shape. The tumor was predominantly composed of elongated cells forming rings around blood vessels(Fig. 1), after the immunohistochemical staining show GFAPID2-40[ S100uVimentin have positive expression, and Ki-67 proliferation index is 1\% 5\%(Fig. 2), FISH-tissue show MYB gene rearrangement detection(Fig. 3). According these evidences, this case was diagnosed as angiocentric glioma. The patient was in good condition after the operation and was discharged one 
month after the operation. After 9 months of follow-up, the patient's physical condition was stable and there was no recurrence.

\section{Result}

\section{CT scan}

CT scan of the patient's brain(Fig.1)

\section{HE staining}

All paraffin-embedded tissues are continuously sliced into slices with a thickness of $4 \mathrm{~cm}$. Regular $\mathrm{HE}$ staining is performed, and the histology is observed under the light microscope(Fig.1)

\section{Immunohistochemical detection}

The immunohistochemical staining was performed using the Envi. si on the two-step method. The antibody GFAP D2-40 QVimentin was purchased from Maixin, China; The antibody S100 was purchased from LBP, China; The antibody Ki67 was purchased from Roche, Switzerland.(Fig.2)

\section{MYB gene rearrangement detection (FISH-tissue)}

Morphological evaluation of tissues and glass slides to determine tumor and hybridization area, using LBP MYB gene break probe to perform fluorescence in situ hybridization, analyze at least two fields of view of 200 tumor cells during division. The probe used this time is a fracture rearrangement probe, provided by Guangzhou KINGMED Center for Clinical Laboratory.(Fig.3)

\section{Discussion}

\section{Histology}

There are a hypotheses: epilepsy-centric methods[3-4]. The epilepsy-centric approach provides evidence that infiltrating the neocortex around tumors is the key to tumor-related epileptic activity, which is due to glioma-related glutamatergic and $y$-aminobutyric acid changes leading to epileptic properties. The neurotransmitter glutamate is used as a "tumor growth factor" by glioma cells. Tumor cells alter the expression of glutamate transporters, including the cystine-glutamate transporter system, and also increase the concentration of extracellular glutamate, leading to seizures, tumor proliferation, and neurotoxicity. According to relevant literature, focal cortical dysplasia has been reported in most cases of $A G[5-8]$, which is similar to other low-grade drug-resistant epilepsy-related tumors (such as ganglioglioma, dysfetal neuroepithelial tumors), that maybe the reason to explain why epilepsy happence with AG. However, in our case, no cortical dysplasia was found, which seems to explain the absence of epilepsy, the above studies are all low-level diagnoses from histopathology, This theory is derived from 
glioma, whether AG-related epilepsy increases the risk of epilepsy due to specific histological features such as the need for more evidence.

\section{2区Molecular Science}

Tumor-centric methods point out that epileptic activity originates from the tumor itself, regarding the interaction of epileptic molecules, and whether there are characteristic molecular mechanisms that cause epilepsy in AG-related epilepsy.

\subsection{MYB-QKI gene}

Some scholars believe that MYB-QKI gene rearrangement is the decisive genetic change of angiocentric glioma tumors[9]. On the one hand, MYB protein is a transcription factor characterized by highly conserved DNA binding motifs, but the C-terminal MYB truncation is carcinogenic, and the MYB-QKI breakpoint in the MYB intron will cause the C-terminal truncation of MYB[10]. On the other hand, MYB-QKI rearrangement destroys the tumor suppressor QKI and promotes tumor formation by reducing the expression of the tumor suppressor gene QKI. The destruction of MYB and QKI seems to promote tumor formation cooperatively. The author[10] claims that compared with normal pediatric brain and PLGG( Pediatric low-grade glioma) without MYB-QKI changes, angiocentric gliomas show significantly higher expression of MYB-QKI characteristics, and MYB-QKI rearrangement seems to be unique to angiocentric gliomas. In addition, it is proposed that the expression of MYB-QKI may lead to the repeated occurrence of intracranial tumors. Therefore, further research on the related mechanism of MYB-QKI gene rearrangement is not only the study of the pathogenic mechanism of AG but also beneficial to the treatment and diagnosis of $A G[11]$.

We used this case without a history of epilepsy to carry out MYB gene rearrangement detection, and found that MYB gene breakage and rearrangement still exist, again verifying that MYB gene breakage and rearrangement is indeed an important factor in the occurrence of AG. However, on the other hand, The MYB gene does not make a difference because of whether there are epileptic seizures, so we boldly guess that MYB gene may not be related to AG-related seizures. At least whether AG patients have epilepsy symptoms, it is not directly related to MYB gene.

\subsection{HCN channel and TRIP8b gene:}

Hyperpolarized activated cyclic nucleotide-gated channel (HCN) can be used as a pacemaker in the brain, becoming a potential breakthrough in the study of epileptic seizures[12]. Studies have confirmed that the sequence variations of HCN1 and HCN2 genes are related to human epilepsy. TPR-containing Rab8b interacting protein [TRIP8b, also known as Pex5p-related protein (PEX5Rp) or H-channel interacting protein 1 (HIP1)], is a brain-specific protein that can bind to Rab8b[13]. Therefore, some scholars believe that TRIP8b is a Potential gene for hereditary epilepsy. And whether epilepsy caused by AG can also seek new attempts in the HCN channel and TRIP8b gene mutations.

\subsection{BRAF V600E mutation}


A recent Meta-analysis article stated: Updates based on a large number of clinical data and a comprehensive Meta-analysis analysis show that BRAF V600E mutation is a specific biomarker[14-15]. This meta-analysis shows that BRAF V600E mutation is involved in epilepsy-related brain tumors (EAT). It plays a key role in the occurrence and development of AG. Are the symptoms of AG epilepsy related to the BRAF V600E mutation? Most scholars now believe that the BRAF V600E mutation is a potential pharmacological target for patients with ganglion glioma, but according to some literature, angiocentric glioma has not been stained by BRAF V600E. Therefore, some scholars believe that positive staining cases are unlikely to be angiocentric gliomas, even BRAF V600E mutation may become one of the exclusion diagnostic criteria for angiocentric gliomas. Based on the above research results, there is no clear evidence showing epilepsy of AG is necessarily related to the BRAF V600E mutation. However, more cases reported about AG will appear and BRAF-positive cases may be found later. Perhaps in certain circumstances, AG may also have some connections with BRAF V600E mutations, and more cases and studies are needed to clarify.

\section{Conclusion}

This report is a case of angiocentric glioma (AG), it is special because this case lack of history of epilepsy, and almost previoce AG case reports observe epilepsy as typical clinical feature. we according CT scanロHistologic findings to diagnose that the case is angiocentric glioma. In order to verify whether the MYB gene is related to AG epilepsy Related, then the MYB gene breakage rearrangement in this case was detected by FISH technology, and this case was compared with most previous AG cases to explore the cause of AG-related seizures. Based on the above evidence, We believe that MYB gene break rearrangement is related to the cause of $A G$ and MYB-QKI gene rearrangement may be one of the important causes of $A G$, but it is not directly related to AG-related epilepsy.

\section{Abbreviations}

AG: angiocentric glioma; HE: Hematoxylin and eosin stain; WHO: World Health Organization; GFAP: glial fibrillary acidic protein; D2-40:Podoplanin Monoclonal Antibody; S100: S100 Polyclonal Antibody; Vimentin: Vimentin Polyclonal Antibody; EMA: Epithelial Membrane Antibody; Ki67: Anti-Ki67 antibody; CT: Computed Tomography; PLGG: Pediatric low-grade glioma; EAT: Epilepsy-related brain tumors;

\section{Declarations}

\section{Authors' contributions}

HLC carried out HE and immunohistochemistry stain and drafted the manuscript. XXS supplied the CT image information. WHY supplied the case of the patient. QQX diagnosed the case. All authors read and approved the final manuscript.

\section{Availability of data and materials}


The original contributions presented in the study are included in the article/ Supplementary Material. Further inquiries can be directed to the corresponding authors.

\section{Ethics approval and consent to paticipate}

The institutional review board of Peking University Shenzhen Hospital approved the study. Written informed consent was obtained from the patient.

\section{Competing interests}

The authors declare that they have no competing interest.

\section{References}

1. Preusser M , Hoischen A, Novak K, et al. Angiocentric Glioma[J]. American Journal of Surgical Pathology, 2007, 31(11):1709-1718.

2. Min W, Tarik T, Rojiani A M, et al. Monomorphous angiocentric glioma: a distinctive epileptogenic neoplasm with features of infiltrating astrocytoma and ependymoma.[J]. Journal of Neuropathology \& Experimental Neurology, 2005(10):875-81.

3. Osborn A G. Angiocentric Neuroepithelial Tumor (ANET): A New Epilepsy-Related Clinicopathological Entity With Distinctive MRI[J]. Yearbook of Diagnostic Radiology, 2006, 2006:371-373.

4. Johan, Pallud, Laurent, et al. Tumoral epileptogenicity: How does it happen?[J]. Epilepsia, 2013, 54(Supplement):30-34.

5. Slegers Rutger Juriaan,Blumcke Ingmar,Low-grade developmental and epilepsy associated brain tumors: a critical update 2020.[J] .Acta Neuropathol Commun, 2020, 8: 27.

6. Preusser M, Hoischen A, Novak K, et al. Angiocentric glioma: report of clinicopathologic and genetic findings in 8 cases.[J]. American Journal of Surgical Pathology, 2007, 31(11):1709.

7. Shakur S F, Mcgirt M J, Johnson M W, et al. Angiocentric glioma: a case series[J]. J Neurosurg Pediatr, 2009, 3(3):197-202.

8. Mott R T, Ellis T L, Geisinger K R. Angiocentric glioma: A case report and review of the literature[J]. Diagnostic Cytopathology, 2010, 38(6):452-456.

9. MYB-QKI rearrangements in angiocentric glioma drive tumorigenicity through a tripartite mechanism[J]. Nature Genetics, 2016.

10. Wefers Annika K,Stichel Damian,Schrimpf Daniel et al. Isomorphic diffuse glioma is a morphologically and molecularly distinct tumour entity with recurrent gene fusions of MYBL1 or MYB and a benign disease course.[J] .Acta Neuropathol, 2020, 139: 193-209.

11. Chan E, Bollen A W, Sirohi D, et al. Angiocentric glioma with MYB-QKI fusion located in the brainstem, rather than cerebral cortex[J]. Acta neuropathologica, 2017, 134(4): 671-673.

12. Reid C A, Phillips A M, Petrou S. HCN channelopathies: pathophysiology in genetic epilepsy and therapeutic implications[J]. Br J Pharmacol, 2011, 165(1):49-56. 
13. Scherschel Katharina,Bräuninger Hanna,Mölders Andrea et al. Characterization of the HCN Interaction Partner TRIP8b/PEX5R in the Intracardiac Nervous System of TRIP8b-Deficient and WildType Mice.[J] .Int J Mol Sci, 2021, 22: undefined.

14. Qi X L,Yao K,Duan Z J et al. [BRAF V600E mutation and clinicopathologic characteristics in 250 cases of brain tumors associated with epilepsy].[J] .Zhonghua Bing Li Xue Za Zhi, 2018, 47: 664-670.

15. Yusoff A A M, Radzak S M A, Nashwa Z, et al. Significance of BRAF V600E Mutation in Intra-axial Brain Tumor in Malaysian Patients: Case Series and Literature Review[J]. Experimental Oncology, 2021, 43(2):2021.

\section{Figures}

\section{CT scan and HE staining}

A

B

C
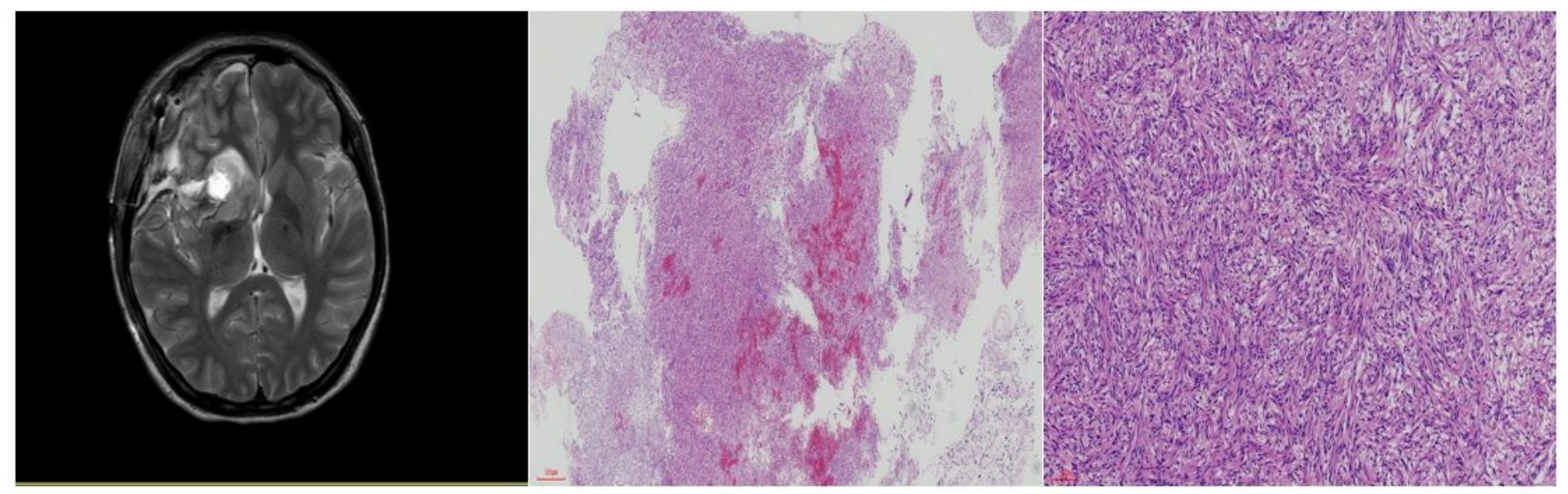

D

E

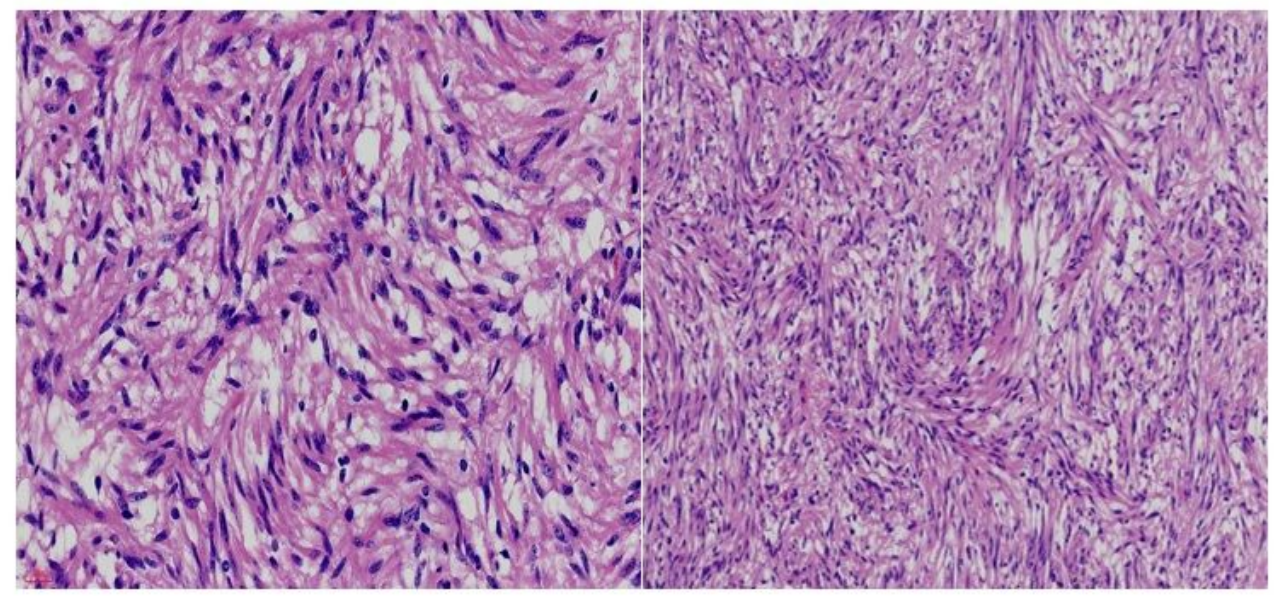

Figure 1 


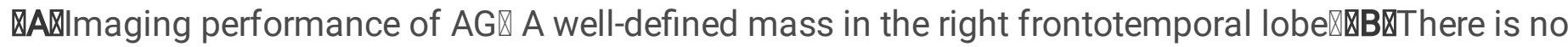

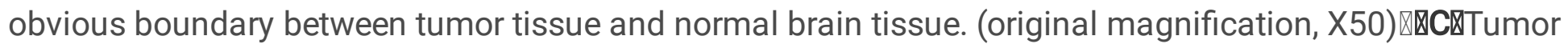
cells are arranged in a water-like and fence-like arrangement (original magnification, X200) QषDQThe tumor

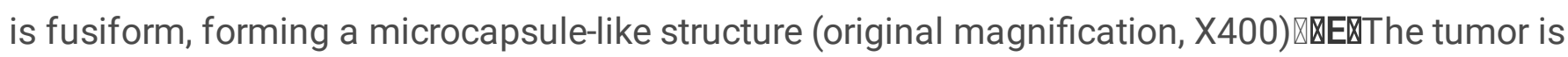
predominantly composed of elongated tumor cells forming rings around the blood vessels (original magnification, X200).

\section{Immunohistochemical detection}
A
B
C

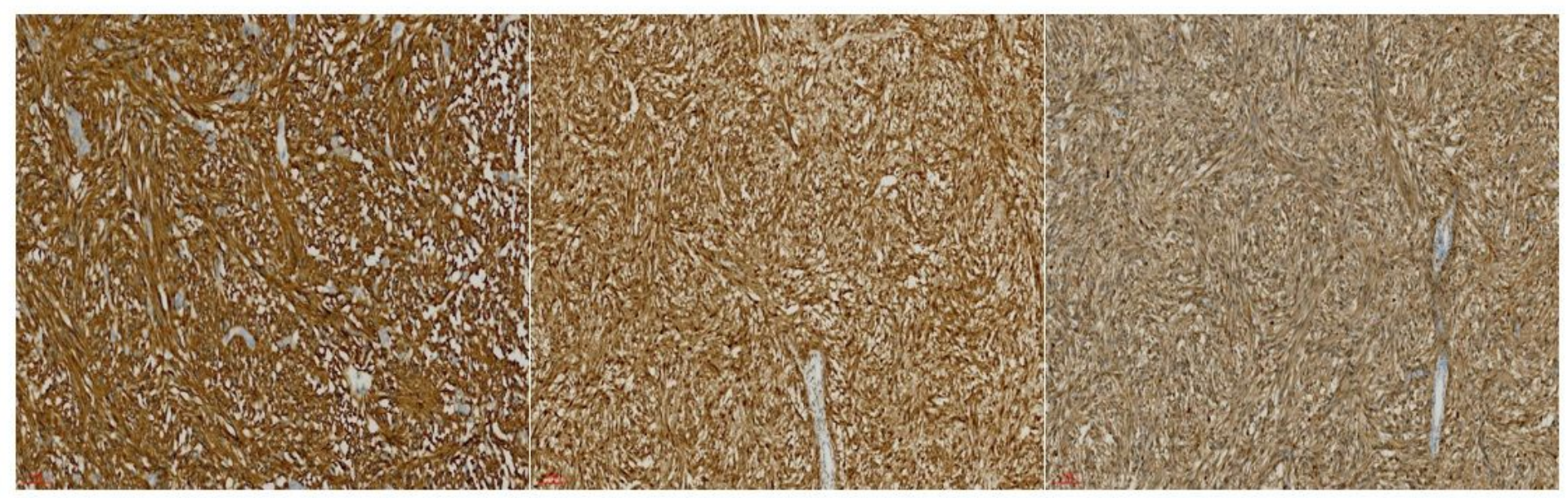

D

E

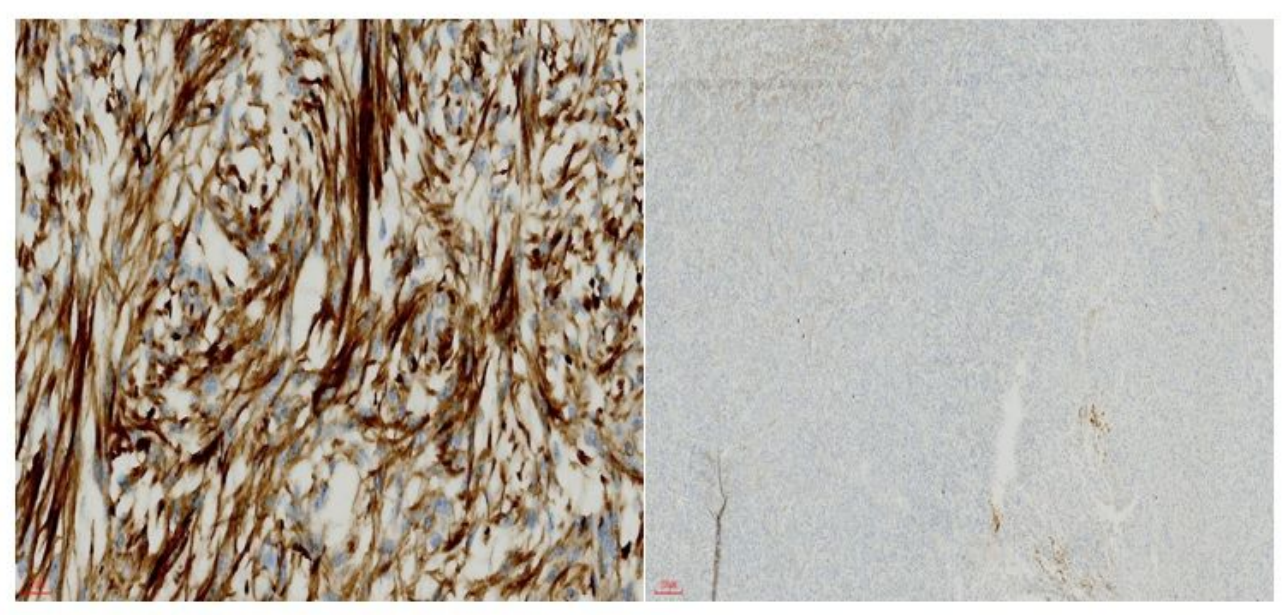

Figure 2

(A) The tumor cells showing variable cytoplasmic immunoreactivity with GFAP (original magnification, X200); (B) The tumor cells showing variable cytoplasmic immunoreactivity with D240(original magnification, X200); (C) The tumor cells showing variable cytoplasmic immunoreactivity with S100 (original magnification, X200); (D) The tumor cells showing variable cytoplasmic immunoreactivity with 


\section{FISH technology detection}

\section{MYB gene break rearrangement test: detected}

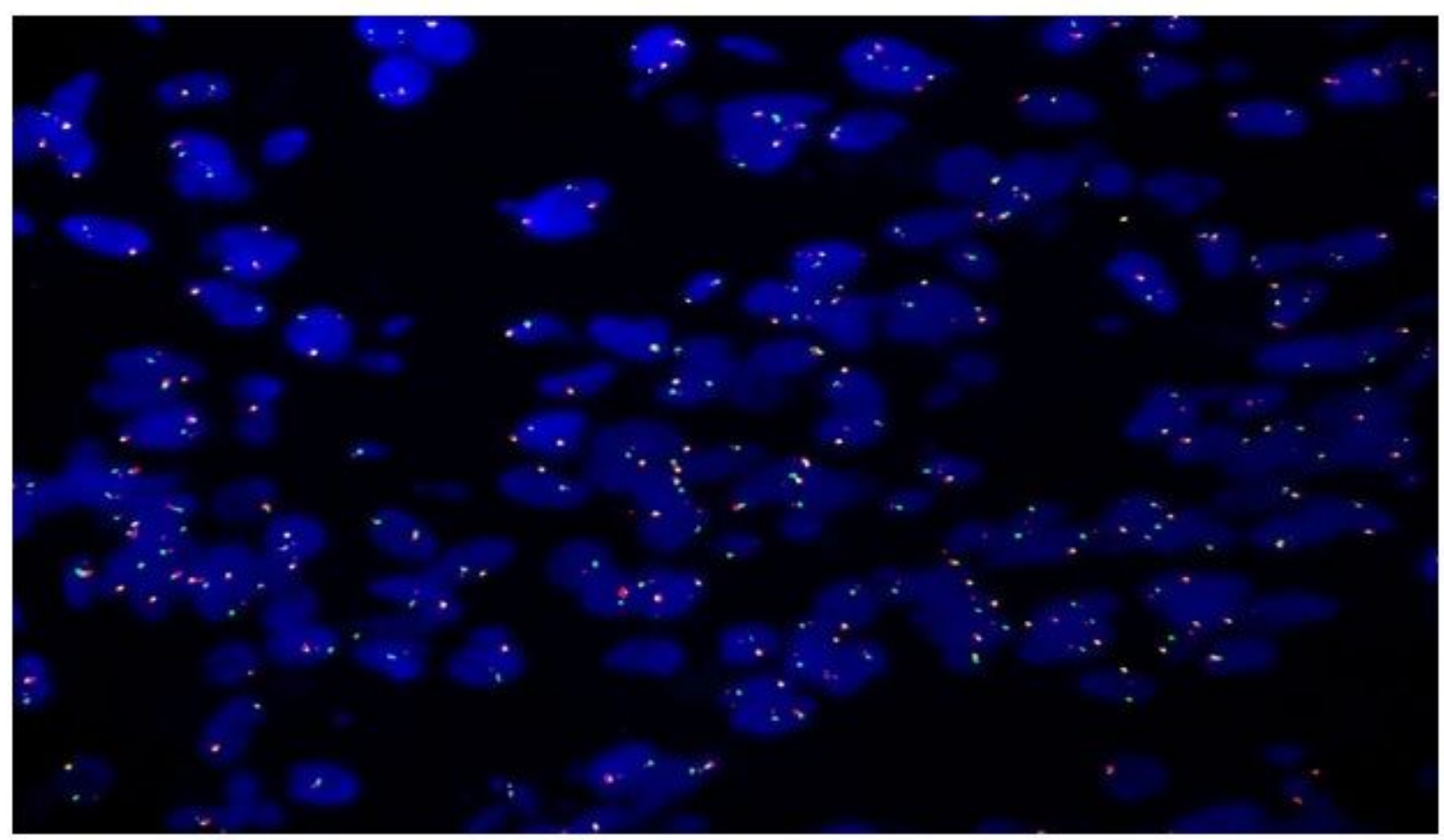

Figure 3

Interpretation:This probe is a fragment rearrangement probe, red fluorescently labeled 5'MYB (6q23) probe, green fluorescently labeled $3^{\prime} \mathrm{MYB}$ probe, normal signal mode is $2 \mathrm{~F}$ (Note: $\mathrm{F}$ is yellow signal or green and red superimposed signal). 